Article

\title{
A Note on the Degenerate Poly-Cauchy Polynomials and Numbers of the Second Kind
}

\author{
Hye Kyung Kim ${ }^{1}$ and Lee-Chae Jang ${ }^{2, *}$ \\ 1 Department of Mathematics Education, Daegu Catholic University, Gyeongsan 38430, Korea; hkkim@cu.ac.kr \\ 2 Graduate School of Education, Konkuk University, Seoul 05029, Korea \\ * Correspondence: Lcjang@konkuk.ac.kr
}

Received: 27 April 2020; Accepted: 5 June 2020; Published: 28 June 2020

check for updates

\begin{abstract}
In this paper, we consider the degenerate Cauchy numbers of the second kind were defined by Kim (2015). By using modified polyexponential functions, first introduced by Kim-Kim (2019), we define the degenerate poly-Cauchy polynomials and numbers of the second kind and investigate some identities and relationship between various polynomials and the degenerate poly-Cauchy polynomials of the second kind. Using this as a basis of further research, we define the degenerate unipoly-Cauchy polynomials of the second kind and illustrate their important identities.
\end{abstract}

Keywords: polylogarithm functions; unipoly functions; Cauchy polynomials; poly-Cauchy polynomials; unipoly-Cauchy polynomials

\section{Introduction}

We first introduce the Cauchy polynomials $C_{n}(x)$ (or the Bernoulli polynomials of the second kind) derived from the integral as follows ( see References [1-4]):

$$
\int_{0}^{1}(1+t)^{x+y} d y=\frac{t}{\log (1+t)}(1+t)^{x}=\sum_{n=0}^{\infty} C_{n}(x) \frac{t^{n}}{n !}
$$

When $x=0, C_{n}=C_{n}(0)$ are called the Cauchy numbers. The Daehee polynomial $D_{n}(x)$ was defined by Kim as the following generating function (see References [5-10]):

$$
\frac{\log (1+t)}{t}(1+t)^{x}=\sum_{n=0}^{\infty} D_{n}(x) \frac{t^{n}}{n !}
$$

When $x=0, D_{n}=D_{n}(0)$ are called the Daehee numbers. Kim [2] defined the degenerate Cauchy polynomials $C_{n, \lambda}(x)$ as follows:

$$
\begin{aligned}
\int_{0}^{1}\left(1+\log (1+\lambda t)^{\frac{1}{\lambda}}\right)^{x+y} d y & =\frac{\frac{1}{\lambda} \log (1+\lambda t)}{\log \left(1+\frac{1}{\lambda} \log (1+\lambda t)\right)}\left(1+\log (1+\lambda t)^{\frac{1}{\lambda}}\right)^{x} \\
& =\sum_{n=0}^{\infty} C_{n, \lambda}(x) \frac{t^{n}}{n !}
\end{aligned}
$$


When $x=0, C_{n, \lambda}=C_{n, \lambda}(0)$ are the degenerate Cauchy numbers. The degenerate Cauchy polynomials $C_{n, \lambda, 2}(x)$ of the second kind are introduced by Kim to be (see References [11-13]):

$$
\frac{t}{\log \left(1+\frac{1}{\lambda} \log (1+\lambda t)\right)}\left(1+\frac{1}{\lambda} \log (1+\lambda t)\right)^{x}=\sum_{n=0}^{\infty} C_{n, \lambda, 2}(x) \frac{t^{n}}{n !},
$$

When $x=0, C_{n, \lambda, 2}=C_{n, \lambda, 2}(0)$ are called the degenerate Cauchy polynomials of the second kind. Here, we note that a major study is to define these polynomials formal with more interesting conditions. From now on, we introduce the following polynomials studied by many researchers to find the identity and relationship between the various polynomials and new defined polynomials. Pyo-Kim-Kim defined the degenerate Cauchy polynomials $C_{n, \lambda, 3}(x)$ of the third kind as follows (see References [11-13]):

$$
\frac{\lambda\left((1+\lambda \log (1+t))^{\frac{1}{\lambda}}-1\right)}{\log (1+\lambda \log (1+t))}(1+\lambda \log (1+t))^{\frac{x}{\lambda}}=\sum_{n=0}^{\infty} C_{n, \lambda, 3}(x) \frac{t^{n}}{n !},
$$

and also defined the degenerate Cauchy polynomials $C_{n, \lambda, 4}(x)$ of the fourth kind as follows (see References [14-16]):

$$
\frac{\lambda t}{\log (1+\lambda \log (1+t))}(1+\lambda \log (1+t))^{\frac{x}{\lambda}}=\sum_{n=0}^{\infty} C_{n, \lambda, 4}(x) \frac{t^{n}}{n !},
$$

Let us introduce the following numbers to find the identity and relationship between various polynomials and new defined polynomials. It is well known that the Stirling numbers of the first kind are defined by (see References $[3,11,17,18]$ ):

$$
(x)_{n}=\sum_{l=0}^{n} S_{1}(n, l) x^{l},
$$

where $(x)_{0}=1,(x)_{n}=x(x-1) \ldots(x-n+1),(n \geq 1)$. From (7), it is easy to see that (see References $[3,17])$ :

$$
\frac{1}{k !}(\log (1+t))^{k}=\sum_{n=k}^{\infty} S_{1}(n, k) \frac{t^{n}}{n !},
$$

In the inverse expression to (7), for $n \geq 0$, the Stirling numbers of the second kind are defined by (see References [4,6,19-24]):

$$
x^{n}=\sum_{l=0}^{n} S_{2}(n, l)(x)_{l},
$$

From (10), it is easy to see that (see References $[17,25]$ ):

$$
\frac{1}{k !}\left(e^{t}-1\right)^{k}=\sum_{n=k}^{\infty} S_{2}(n, k) \frac{t^{n}}{n !}
$$

In this paper, we consider the degenerate Cauchy numbers of the second kind were defined by Kim (2015). By using modified polyexponential functions, first introduced by Kim-Kim (2019), we define the degenerate poly-Cauchy polynomials and numbers of the second kind and investigate some identities and relationship between various polynomials and the degenerate poly-Cauchy polynomials of the second kind. Using this as a basis of further research, we define the degenerate unipoly-Cauchy polynomials of the second kind and illustrate their important identities. 


\section{The Degenerate Poly-Cauchy Polynomials of the Second Kind}

For $k \in \mathbb{Z}$, it is well known that the polylogarithm function $L i_{k}(x)$ is defined by a power series in $x$ to be (see References $[8,18,19,26])$ :

$$
\operatorname{Li}_{k}(x)=\sum_{n=1}^{\infty} \frac{x^{n}}{n^{k}}=x+\frac{x^{2}}{2^{k}}+\frac{x^{3}}{3^{k}}+\cdots,
$$

The polyexponential function was first studied by Hardy and then the polyexponential function modified by Kim-Kim was studied (see Reference [15]). Recently, Kim-Kim [19] considered the polyexponential function in the view of an inverse type of the polylogarithm function to be

$$
E i_{k}(x)=\sum_{n=1}^{\infty} \frac{x^{n}}{(n-1) ! n^{k}}
$$

In (12), when $k=1$, we get $E i_{1}(x)=e^{x}-1$. By using the modified polyexponential function, they also introduced type 2 poly-Bernoulli polynomials and unipoly-Bernoulli polynomials [19] .

In the same motivation of type 2 poly-Bernoulli polynomials arising from modified polyexponential function, we define the degenerate poly-Cauchy polynomials of the second kind as follows:

$$
\frac{E i_{k}(\log (1+t))}{\log \left(1+\frac{1}{\lambda} \log (1+\lambda t)\right)}\left(1+\frac{1}{\lambda} \log (1+\lambda t)\right)^{x}=\sum_{n=0}^{\infty} C_{n, \lambda, 2}^{(k)}(x) \frac{t^{n}}{n !} .
$$

When $x=0, C_{n, \lambda, 2}^{(k)}=C_{n, \lambda, 2}^{(k)}(0)$ are called the degenerate poly-Cauchy numbers of the second kind. For $k=1$, by (12), we note that

$$
\begin{aligned}
E i_{1}(\log (1+t)) & =\sum_{n=1}^{\infty} \frac{(\log (1+t))^{n}}{(n-1) ! n} \\
& =e^{\log (1+t)}-1=t
\end{aligned}
$$


By (13) and (14), we see that $C_{n, \lambda, 2}^{(1)}=C_{n, \lambda, 2}$. From (13) with $x=0$, we observe that

$$
\begin{aligned}
& \sum_{n=0}^{\infty} C_{n, \lambda, 2}^{(k)} \frac{t^{n}}{n !}=\frac{E i_{k}(\log (1+t))}{\log \left(1+\frac{1}{\lambda} \log (1+\lambda t)\right)} \\
& =\frac{t}{\log \left(1+\frac{1}{\lambda} \log (1+\lambda t)\right)} \frac{1}{t} \sum_{m=1}^{\infty} \frac{(\log (1+t))^{m}}{(m-1) ! m^{k}} \\
& =\frac{t}{\log \left(1+\frac{1}{\lambda} \log (1+\lambda t)\right)} \frac{1}{t} \sum_{m=0}^{\infty} \frac{(\log (1+t))^{m+1}}{(m+1) !(m+1)^{k-1}} \\
& =\frac{t}{\log \left(1+\frac{1}{\lambda} \log (1+\lambda t)\right)} \frac{1}{t} \sum_{m=0}^{\infty} \frac{1}{(m+1)^{k-1}} \sum_{l=m+1}^{\infty} S_{1}(l, m+1) \frac{t^{l}}{l !} \\
& =\frac{t}{\log \left(1+\frac{1}{\lambda} \log (1+\lambda t)\right)} \sum_{m=0}^{\infty} \frac{1}{(m+1)^{k-1}} \sum_{l=m}^{\infty} S_{1}(l+1, m+1) \frac{t^{l}}{(l+1) !} \\
& =\left(\sum_{s=0}^{\infty} C_{s, \lambda, 2} \frac{t^{s}}{s !}\right)\left(\sum_{l=0}^{\infty} \sum_{m=0}^{l} \frac{1}{(m+1)^{k-1}} \frac{S_{1}(l+1, m+1)}{l+1} \frac{t^{l}}{l !}\right) \\
& =\sum_{n=0}^{\infty}\left(\sum_{l=0}^{n} \sum_{m=0}^{l}\left(\begin{array}{l}
n \\
l
\end{array}\right) C_{n-l, \lambda, 2} \frac{S_{1}(l+1, m+1)}{(l+1)(m+1)^{k-1}}\right) \frac{t^{n}}{n !} .
\end{aligned}
$$

Therefore, by (15), we obtain the following theorem which is an identity between the Stirling numbers of the first kind and the degenerate poly-Cauchy numbers of the second kind.

Theorem 1. For $n \geq 0, k \in \mathbb{Z}$, we have

$$
C_{n, \lambda, 2}^{(k)}=\sum_{l=0}^{n} \sum_{m=0}^{l}\left(\begin{array}{l}
n \\
l
\end{array}\right) C_{n-l, \lambda, 2} \frac{S_{1}(l+1, m+1)}{(l+1)\left((m+1)^{k-1}\right.} .
$$

Let us take $k=1$. From (16), we get a very interesting recurrence relation as follows.

$$
\sum_{l=1}^{n} \sum_{m=0}^{l}\left(\begin{array}{l}
n \\
l
\end{array}\right) C_{n-l, \lambda, 2} \frac{S_{1}(l+1, m+1)}{(l+1)}=0
$$

In Reference [19], it is well known that

$$
\frac{d}{d x} E i_{k}(\log (1+x))=\frac{1}{(1+x) \log (1+x)} E i_{k-1}(\log (1+x)) .
$$

From (18), we note that

$$
\begin{aligned}
& E i_{k}(\log (1+x))=\int_{0}^{x} \frac{1}{(1+t) \log (1+t)} E i_{k-1}(\log (1+t)) d t \\
& =\int_{0}^{x} \frac{1}{(1+t) \log (1+t)} \underbrace{\int_{0}^{t} \frac{1}{(1+t) \log (1+t)} \int_{0}^{t} \cdots \int_{0}^{t} \frac{t}{(1+t) \log (1+t)} d t d t \cdots d t .}_{(k-2) \text { times }}
\end{aligned}
$$


It is well known that

$$
\frac{t}{(1+t) \log (1+t)}=\sum_{l=0}^{\infty} B_{l}^{(l)} \frac{t^{l}}{l !}
$$

The above formula is very important and is used at the core of finding the relational formula. From (14), (19), and (20), we can find the following relational formula.

$$
\begin{aligned}
& \sum_{n=0}^{\infty} C_{n, \lambda, 2}^{(k)} \frac{x^{n}}{n !}=\frac{E i_{k}(\log (1+x))}{\log \left(1+\frac{1}{\lambda} \log (1+\lambda x)\right)} \\
& =\frac{1}{\log \left(1+\frac{1}{\lambda} \log (1+\lambda x)\right)} \int_{0}^{x} \frac{1}{(1+t) \log (1+t)} E i_{k-1}(\log (1+t)) d t \\
& =\frac{1}{\log \left(1+\frac{1}{\lambda} \log (1+\lambda x)\right)} \int_{0}^{x} \frac{1}{(1+t) \log (1+t)} \\
& \quad \times \underbrace{\int_{0}^{t} \frac{1}{(1+t) \log (1+t)} \int_{0}^{t} \cdots \int_{0}^{t}}_{(k-2) \text { times }} \frac{t}{(1+t) \log (1+t)} d t \cdots d t d t .
\end{aligned}
$$

Let us take $k=2$. Then we can find a clearer case relationship in Equation (21)

$$
\begin{aligned}
& \sum_{n=0}^{\infty} C_{n, \lambda, 2}^{(2)} \frac{t^{n}}{n !} \\
& =\frac{1}{\log \left(1+\frac{1}{\lambda} \log (1+\lambda x)\right)} \int_{0}^{x} \frac{t}{(1+t) \log (1+t)} d t \\
& =\frac{1}{\log \left(1+\frac{1}{\lambda} \log (1+\lambda x)\right)} \sum_{l=0}^{\infty} \frac{B_{l}^{(l)}}{l !} \int_{0}^{x} t^{l} d t \\
& =\frac{x}{\log \left(1+\frac{1}{\lambda} \log (1+\lambda x)\right)} \sum_{l=0}^{\infty} \frac{B_{l}^{(l)}}{l+1} \frac{x^{l}}{l !} \\
& =\left(\sum_{m=0}^{\infty} C_{m, \lambda, 2} \frac{x^{m}}{m !}\right)\left(\sum_{l=0}^{\infty} \frac{B_{l}^{(l)}}{l+1} \frac{x^{l}}{l !}\right) \\
& =\sum_{n=0}^{\infty}\left(\sum_{l=0}^{n}\left(\begin{array}{l}
n \\
l
\end{array}\right) \frac{C_{n-l, \lambda, 2} B_{l}^{(l)}}{l+1}\right) \frac{x^{n}}{n !} .
\end{aligned}
$$

Therefore, by (22), we found an identity equation that could calculate the degenerate poly-Cauchy numbers of the second kind from the degenerate Cauchy numbers of the second kind when $k=2$ as the following theorem.

Theorem 2. Let $n \geq 0$. Then we have

$$
C_{n, \lambda, 2}^{(2)}=\sum_{l=0}^{n}\left(\begin{array}{l}
n \\
l
\end{array}\right) \frac{C_{n-l, \lambda, 2} B_{l}^{(l)}}{l+1} .
$$


From (13), we observe that

$$
\begin{aligned}
& \sum_{n=0}^{\infty} C_{n, \lambda, 2}^{(k)}(x) \frac{t^{n}}{n !} \\
& =\frac{E i_{k}(\log (1+t))}{\log \left(1+\frac{1}{\lambda} \log (1+\lambda t)\right)}\left(1+\frac{1}{\lambda} \log (1+\lambda t)\right)^{x} \\
& =\left(\sum_{l=0}^{\infty} C_{l, \lambda, 2}^{(k)} \frac{t^{l}}{l !}\right)\left(\sum_{m=0}^{\infty}\left(\begin{array}{c}
x \\
m
\end{array}\right)\left(\frac{1}{\lambda} \log (1+\lambda t)\right)^{m}\right) \\
& =\left(\sum_{l=0}^{\infty} C_{l, \lambda, 2}^{(k)} \frac{t^{l}}{l !}\right)\left(\sum_{m=0}^{\infty}(x)_{m} \lambda^{-m} \sum_{s=m}^{\infty} S_{1}(s, m) \lambda^{s} \frac{t^{s}}{s !}\right) \\
& =\left(\sum_{l=0}^{\infty} C_{l, \lambda, 2}^{(k)} \frac{t^{l}}{l !}\right)\left(\sum_{s=0}^{\infty} \sum_{m=0}^{s}(x)_{m} \lambda^{-m} S_{1}(s, m) \lambda^{s} \frac{t^{s}}{s !}\right) \\
& =\sum_{n=0}^{\infty}\left(\sum_{l=0}^{n} \sum_{m=0}^{n-l}\left(\begin{array}{l}
n \\
l
\end{array}\right) C_{l, \lambda, 2}^{(k)}(x)_{m} \lambda^{n-l-m} S_{1}(n-l, m)\right) \frac{t^{n}}{n !} .
\end{aligned}
$$

By comparing the coefficients on both sides of (24), we found an recurrence relation between the degenerate poly-Cauchy polynomials of the second kind as the following theorem.

Theorem 3. Let $n \geq 0$ and $k \in \mathbb{Z}$. Then we have

$$
C_{n, \lambda, 2}^{(k)}(x)=\sum_{l=0}^{n} \sum_{m=0}^{n-l}\left(\begin{array}{l}
n \\
l
\end{array}\right) C_{l, \lambda, 2}^{(k)}(x)_{m} \lambda^{n-l-m} S_{1}(n-l, m) .
$$

\section{The Degenerate Unipoly-Cauchy Polynomials of the Second Kind}

Let $p(n)$ be any arithmetic function which is a real or complex valued function defined on the set of positive integers $\mathbb{N}$. Then Kim-Kim [19] defined the unipoly function attached to $p$ by

$$
u_{k}(x \mid p)=\sum_{n=1}^{\infty} \frac{p(n) x^{n}}{n^{k}}, \quad(k \in \mathbb{Z}) .
$$

It is well known that

$$
u_{k}(x \mid 1)=\sum_{n=1}^{\infty} \frac{x^{n}}{n^{k}}=L i_{k}(x)
$$

is ordinary polylogarithm function, and for $k \geq 2$,

$$
\frac{d}{d x} u_{k}(x \mid p)=\frac{1}{x} u_{k-1}(x \mid p),
$$

and

$$
u_{k}(x \mid p)=\int_{0}^{x} \frac{1}{t} \underbrace{\int_{0}^{t} \cdots \int_{0}^{t}}_{(k-2) \text { times }} \frac{1}{t} u_{1}(t \mid p) d t \cdots d t d t \text {, (see Reference [19]). }
$$


By using (26), we define the degenerate unipoly-Cauchy polynomials of the second kind as follows:

$$
\frac{u_{k}(\log (1+t) \mid p)}{\log \left(1+\frac{1}{\lambda} \log (1+\lambda t)\right)}\left(1+\frac{1}{\lambda} \log (1+\lambda t)\right)^{\frac{x}{\lambda}}=\sum_{n=0}^{\infty} C_{n, \lambda, 2}^{(k, p)}(x) \frac{t^{n}}{n !} .
$$

When $x=0, C_{n, \lambda, 2}^{(k, p)}=C_{n, \lambda, 2}^{(k, p)}(0)$ are called the degenerate unipoly-Cauchy numbers of the second kind. Let us take $p(n)=\frac{1}{\Gamma(n)}$. Then we have

$$
\begin{aligned}
& \sum_{n=0}^{\infty} C_{n, \lambda, 2}^{(k, p)}(x) \frac{t^{n}}{n !}=\frac{u_{k}\left(\log (1+t) \mid \frac{1}{\Gamma}\right)}{\log \left(1+\frac{1}{\lambda} \log (1+\lambda t)\right)}\left(1+\frac{1}{\lambda} \log (1+\lambda t)\right)^{\frac{x}{\lambda}} \\
& =\frac{1}{\log \left(1+\frac{1}{\lambda} \log (1+\lambda t)\right)} \sum_{m=1}^{\infty} \frac{(\log (1+t))^{m}}{m^{k}(m-1) !}\left(1+\frac{1}{\lambda} \log (1+\lambda t)\right)^{\frac{x}{\lambda}} \\
& =\frac{E i_{k}(\log (1+t))}{\log \left(1+\frac{1}{\lambda} \log (1+\lambda t)\right)}\left(1+\frac{1}{\lambda} \log (1+\lambda t)\right)^{\frac{x}{\lambda}} \\
& =\sum_{n=0}^{\infty} C_{n, \lambda, 2}^{(k)}(x) \frac{t^{n}}{n !} .
\end{aligned}
$$

Thus, by (31), we have the following theorem.

Theorem 4. Let $n \geq 0$ and $k \in \mathbb{Z}$, and $\Gamma(n)$ be the Gamma function. Then, we have

$$
C_{n, \lambda, 2}^{\left(k, \frac{1}{\Gamma}\right)}(x)=C_{n, \lambda, 2}^{(k)}(x) .
$$

From (30), we get

$$
\begin{aligned}
& \sum_{n=0}^{\infty} C_{n, \lambda, 2}^{(k, p)} \frac{t^{n}}{n !}=\frac{u_{k}(\log (1+t) \mid p)}{\log \left(1+\frac{1}{\lambda} \log (1+\lambda t)\right)} \\
& =\frac{1}{\log \left(1+\frac{1}{\lambda} \log (1+\lambda t)\right)} \sum_{m=1}^{\infty} \frac{p(m)}{m^{k}(\log (1+t))^{m}} \\
& =\frac{1}{\log \left(1+\frac{1}{\lambda} \log (1+\lambda t)\right)} \sum_{m=0}^{\infty} \frac{p(m+1)(m+1) !}{(m+1)^{k}} \sum_{l=m+1}^{\infty} S_{1}(m+1, l) \frac{t^{l}}{l !} \\
& =\left(\sum_{j=0}^{\infty} C_{j, \lambda, 2} \frac{t^{j}}{j !}\right)\left(\sum_{l=0}^{\infty} \sum_{m=0}^{l} \frac{p(m+1)(m+1) !}{(m+1)^{k}} S_{1}(m+1, l) \frac{t^{l}}{l !}\right) \\
& =\sum_{n=0}^{\infty}\left(\sum_{l=0}^{\infty} \sum_{m=0}^{l}\left(\begin{array}{l}
n \\
l
\end{array}\right) \frac{p(m+1)(m+1) !}{(m+1)^{k}} \frac{S_{1}(m+1, l) C_{n-l, \lambda, 2}}{l+1}\right) \frac{t^{n}}{n !} .
\end{aligned}
$$

Therefore, by comparing the coefficients on both sides of (33), we found an recurrence relation between the degenerate unipoly-Cauchy numbers of the second kind as the following theorem. 
Theorem 5. Let $n \in \mathbb{N}$ and $k \in \mathbb{Z}$. Then we have

$$
C_{n, \lambda, 2}^{(k, p)}=\sum_{l=0}^{\infty} \sum_{m=0}^{l}\left(\begin{array}{l}
n \\
l
\end{array}\right) \frac{p(m+1)(m+1) !}{(m+1)^{k}} \frac{S_{1}(m+1, l) C_{n-l, \lambda, 2}}{l+1} .
$$

In particular,

$$
C_{n, \lambda, 2}^{\left(k, \frac{1}{\Gamma}\right)}=C_{n, \lambda, 2}^{(k)}=\sum_{l=0}^{\infty} \sum_{m=0}^{l}\left(\begin{array}{l}
n \\
l
\end{array}\right) \frac{S_{1}(m+1, l) C_{n-l, \lambda, 2}}{(m+1)^{k-1}(l+1)}
$$

From (30), we observe that

$$
\begin{aligned}
& \sum_{n=0}^{\infty} C_{n, \lambda, 2}^{(k, p)}(x) \frac{t^{n}}{n !}=\frac{u_{k}(\log (1+t) \mid p)}{\log \left(1+\frac{1}{\lambda} \log (1+\lambda t)\right)}\left(1+\frac{1}{\lambda} \log (1+\lambda t)\right)^{\frac{x}{\lambda}} \\
& =\frac{u_{k}(\log (1+t) \mid p)}{\log \left(1+\frac{1}{\lambda} \log (1+\lambda t)\right)} \sum_{m=0}^{\infty}\left(\begin{array}{c}
\frac{x}{\lambda} \\
m
\end{array}\right)\left(\frac{1}{\lambda} \log (1+\lambda t)\right)^{m} \\
& =\left(\sum_{l=0}^{\infty} C_{l, \lambda, 2}^{(k, p)} \frac{t^{l}}{l !}\right)\left(\sum_{m=0}^{\infty}(x)_{m, \lambda} \lambda^{-2 m} \sum_{s=m}^{\infty} S_{1}(s, m) \frac{t^{s}}{s !}\right) \\
& =\left(\sum_{l=0}^{\infty} C_{l, \lambda, 2}^{(k, p)} \frac{t^{l}}{l !}\right)\left(\sum_{s=0}^{\infty} \sum_{m=0}^{s}(x)_{m, \lambda} \lambda^{-2 m} S_{1}(s, m) \frac{t^{s}}{s !}\right) \\
& =\sum_{n=0}^{\infty}\left(\sum_{l=0}^{n} \sum_{m=0}^{n-l} C_{l, \lambda, 2}^{(k, p)}(x)_{m, \lambda} \lambda^{-2 m} S_{1}(n-l, m)\right) \frac{t^{n}}{n !} .
\end{aligned}
$$

From (36), we found an recurrence relation between the degenerate unipoly-Cauchy polynomials of the second kind as the following theorem.

Theorem 6. Let $n \geq 0$ and $k \in \mathbb{Z}$. Then we have

$$
C_{n, \lambda, 2}^{(k, p)}(x)=\sum_{l=0}^{n} \sum_{m=0}^{n-l} C_{l, \lambda, 2}^{(k, p)}(x)_{m, \lambda} \lambda^{-2 m} S_{1}(n-l, m) .
$$


From (30), we observe that

$$
\begin{aligned}
\sum_{n=0}^{\infty} C_{n, \lambda, 2}^{(k, p)} \frac{t^{n}}{n !} & \frac{u_{k}(\log (1+t) \mid p)}{\log \left(1+\frac{1}{\lambda} \log (1+\lambda t)\right)} \\
= & \frac{1}{\log \left(1+\frac{1}{\lambda} \log (1+\lambda t)\right)} \sum_{m=0}^{\infty} \frac{p(m+1)}{(m+1)^{k}} \frac{m !}{m !}(\log (1+t))^{m+1} \\
= & \frac{\log (1+t)}{\log \left(1+\frac{1}{\lambda} \log (1+\lambda t)\right)} \sum_{m=0}^{\infty} \frac{p(m+1)}{(m+1)^{k}} \frac{m !}{m !}(\log (1+t))^{m} \\
= & \frac{\log (1+t)}{\log \left(1+\frac{1}{\lambda} \log (1+\lambda t)\right)} \sum_{m=0}^{\infty} \frac{p(m+1)}{(m+1)^{k}} \frac{m !}{m !}(\log (1+t))^{m} \\
= & \frac{\log (1+t)}{t} \frac{t}{\log \left(1+\frac{1}{\lambda} \log (1+\lambda t)\right)} \sum_{m=0}^{\infty} \frac{p(m+1) m !}{(m+1)^{k}} \sum_{l=m}^{\infty} S_{1}(l, m) \frac{t^{l}}{l !} \\
= & \left(\sum_{s=0}^{\infty} D_{s} \frac{t^{s}}{s !}\right)\left(\sum_{a=0}^{\infty} C_{a, \lambda, 2} \frac{t^{a}}{a !}\right)\left(\sum_{l=0}^{\infty} \sum_{m=0}^{l} \frac{p(m+1) m !}{(m+1)^{k}} S_{1}(l, m) \frac{t^{l}}{l !}\right) \\
= & \left(\sum_{b=0}^{\infty} \sum_{a=0}^{b}\left(\begin{array}{l}
b \\
a
\end{array}\right) D_{b-a} C_{a, \lambda, 2} \frac{t^{b}}{b !}\right)\left(\sum_{l=0}^{\infty} \sum_{m=0}^{l} \frac{p(m+1) m !}{(m+1)^{k}} S_{1}(l, m) \frac{t^{l}}{l !}\right) \\
= & \sum_{n=0}^{\infty}\left(\sum_{l=0}^{n} \sum_{a=0}^{n-l} \sum_{m=0}^{l}\left(\begin{array}{l}
n \\
l
\end{array}\right) D_{n-l-a} C_{a, \lambda, 2} \frac{p(m+1) m !}{(m+1)^{k}} S_{1}(l, m)\right) \frac{t^{n}}{n !} .
\end{aligned}
$$

By comparing coefficients on both sides of (38), we obtain the following theorem which is a relationship between Daehee numbers and the degenerate unipoly-Cauchy numbers of the second kind.

Theorem 7. Let $n \geq 0$ and $k \in \mathbb{Z}$. Then we have

$$
C_{n, \lambda, 2}^{(k, p)}=\sum_{l=0}^{n} \sum_{a=0}^{n-l} \sum_{m=0}^{l}\left(\begin{array}{l}
n \\
l
\end{array}\right) D_{n-l-a} C_{a, \lambda, 2} \frac{p(m+1) m !}{(m+1)^{k}} S_{1}(l . m)
$$

\section{Conclusions}

In 2019 Kim-Kim considered the polyexponential functions and poly-Bernoulli polynomials and Kim [2] introduced the degenerate Cauchy numbers of the second kind. In the same view as these functions and polynomials, we defined the degenerate poly-Cauchy polynomials of the second kind Equation (13) and obtained some identities of the degenerate poly-Cauchy numbers of the second kind (Theorems 1 and 2 ). In particular, we obtained an identity of the degenerate poly-Cauchy polynomials of the second kind in Theorem 3. Furthermore, by using the unipoly functions, we defined the degenerate unipoly-Cauchy polynomials of the second kind Equation (30) and obtained some properties of the degenerate unipoly-Cauchy numbers of the second kind (Theorems 4 and 5). Finally, we obtained an identity of the degenerate unipoly-Cauchy polynomials of the second kind in Theorem 6 and gave the identity indicating the relationship of the degenerate unipoly-Cauchy numbers of the second kind and the Daehee numbers and degenerate Cauchy numbers of the second kind in Theorem 7. In Bayad-Hamahata studied the multi-poly-Bernoulli polynomials [27], defined in analogy with the poly-case, using instead 
the multiple polylogarithm functions. In addition, as a good application of the results of this paper, we would recommend readers to see to references [28-30].

Author Contributions: L.-C.J. conceived of the framework and structured the whole paper; H.K.K. checked the results of the paper and completed the revision of the article. All authors have read and agreed to the published version of the manuscript.

Funding: This research is supported by the Basic Science Research Program, the National Research Foundation of Korea, the Ministry of Education, (NRF-2018R1D1A1B07049584).

Acknowledgments: We thank the reviewers for their valuable comments and suggestions and the authors would like to thank the Jacob Wood (jacob.wood@jcu.edu.au) from James Cook University Singapore, a native speaker, for valuable comments to help improve the English sentence of the original manuscript.

Conflicts of Interest: The authors declare that they have no competing interests.

\section{References}

1. Borisov, B.S. The p-binomial transform Cauchy numbers and figurate numbers. Proc. Jangjeon Math. Soc. 2016, 19, 631-644.

2. Kim, T. Degenerate Cauchy numbers and polynomials of the second kind. Adv. Stud. Contemp. Math. 2017, 27, 441-449.

3. Kim, T. On degenerate Cauchy numbers and polynomials. Proc. Jangjeon Math. Soc. 2015, 18, 307-312.

4. Kim, T.; Jang, L.C.; Kim, D.S.; Kim, H.Y. Some identities on type 2 degenerate Bernoulli polynomials of the second kind. Symmetry 2020, 12, 510. [CrossRef]

5. Chung, S.-K.; Jang, G.-W.; Kim, D.S.; Kwon, J. Some identities of the type 2 degenerate Bernoulli and Euler numbers. Adv. Stud. Contemp. Math. 2019, 29, 613-632.

6. Jang, L.C.; Kim, W.; Kwon, H.-I.; Kim, T. On degenerate Daehee polynomials and numbers of the third kind. J. Comput. Appl. Math. 2020, 364, 112343. [CrossRef]

7. Kim, T.; Kim, D.S.; Kim, H.Y.; Jang, L.C. Degenerate poly-Bernoulli numbers and polynomials. Informatica 2020, $31,2-8$.

8. Kim, T.; Kim, D.S.; Kwon, J.; Lee, H. Degenerate polyexponential functions and type 2 degenerate poly-Bernoulli numbers and polynomials. Adv. Differ. Equ. 2020, 2020, 168. [CrossRef]

9. Kwon, J.; Kim, T.; Kim, D.S.; Kim, H.Y. Some identities for degenerate complete and incomplete $r$-Bell polynomials. J. Inequal. Appl. 2020, 2020, 23. [CrossRef]

10. Lim, D. Modified degenerate Daehee numbers and polynomials arising from differential equations. Adv. Stud. Contemp. Math. 2018, 28, 497-506.

11. Kim, T.; Kim, D.S.; Kwon, J.; Lee, H. A note on degenerate gamma random variables. Rev. Educ. 2020, 388, 39-44.

12. Kim, T.; Kim, D.S. Some identities of extended degenerate $r$-central Bell polynomials arising from umbral calculus. Rev. R. Acad. Cienc. Exactas Fis. Nat. Ser. A Mat. RACSAM 2020, 114, 19. [CrossRef]

13. Pyo, S.-S.; Kim, T.; Rim, S.-H. Degenerate Cauchy numbers of the third kind. J. Inequal. Appl. 2018, 32, 12. [CrossRef] [PubMed]

14. Kim, T.; Kim, D.S.; Lee, H.; Kwon, J. Degenerate binomial coefficients and degenerate hypergeometric functions. Adv. Differ. Equ. 2020, 2020, 115. [CrossRef]

15. Kim, T.; Kim, D.S. Degenerate polyexponential functions and degenerate Bell polynomials. J. Math. Anal. Appl. 2020, 487, 124017. [CrossRef]

16. Pyo, S.-S. Degenerate Cauchy numbers and polynomials of the fourth kind. Adv. Stud. Contemp. Math. 2018, 28, 127-138.

17. Duran, U.; Acikgoz, M.; Araci, S. Hermite based poly-Bernoulli polynomials with a q-parameter. Adv. Stud. Contemp. Math. 2018, 28, 285-296.

18. Kim, D.S.; Kim, T. On sums of finite products of balancing polynomials. J. Comput. Appl. Math. 2020, 377, 112913. [CrossRef] 
19. Kim, D.S.; Kim, T. A note on polyexponential and unipoly functions. Russ. J. Math. Phys. 2019, 26, 40-49. [CrossRef]

20. Kim, T.; Kim, D.S.; Dolgy, D.V.; Kwon, J. Some identities on generalized degenerate Genocchi and Euler numbers. Informatica 2020, 31, 42-51.

21. Kim, T.; Kim, D.S.; Jang, L.C.; Kim, H.Y. A note on discrete degenerate random variables. Proc. Jangjeon Math. Soc. 2020, 23, 125-135.

22. Khan, W.A.; Ahmad, M. Partially degenerate poly-Bernoulli polynomials associated with Hermite polynomials. Adv. Stud. Contemp. Math. 2018, 28, 487-496.

23. Kruchinin, D.V.; Kruchinin, V.V. Explicit formula for reciprocal generating function and its application. Adv. Stud. Contemp. Math. 2019, 29, 365-372.

24. Kim, T.; Kim, D.S.; Kwon, J.; Kim, H.Y. A note on degenerate Genocchi and poly-Genocchi numbers and polynomials. J. Inequal. Appl. 2020, 2020, 110. [CrossRef]

25. Roman, S. The umbral calculus. In Pure and Applied Mathematics; Academic Press, Inc.: New York, NY, USA, 1984; p. 193. ISBN 0-12-594380-6.

26. Kaneko, M. Poly-Bernoulli numbers. J. Theor. Nr. Bordeanes 1997, 9, 221-228. [CrossRef]

27. Bayad, A.; Hamahata, Y. Multiple polylogarithms and multi-poly-Bernoulli polynomials. Funct. Approx. Comment. Math. 2012, 46, 45-61. [CrossRef]

28. Kim, T.; Kim, D.S.; Jang, L.-C.; Lee, H. Jindalrae and Gaenari numbers and polynomials in connection with Jindalrae-Stirling numbers. Adv. Differ. Equ. 2020, 2020, 245. [CrossRef]

29. Kim, T.; Kim, D.S. Some relations of two type 2 polynomials and discrete harmonic numbers and polynomials. Symmetry 2020, 12, 905. [CrossRef]

30. Kim, D.S.; Kim, T. A note on a new type of degenerate Bernoulli Numbers. Russ. J. Math. Phys. 2020, 27, $227-235$. [CrossRef]

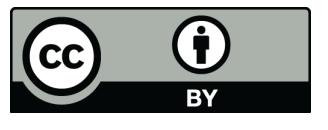

(C) 2020 by the authors. Licensee MDPI, Basel, Switzerland. This article is an open access article distributed under the terms and conditions of the Creative Commons Attribution (CC BY) license (http:/ / creativecommons.org/licenses/by/4.0/). 